\title{
Application and Research of Multimedia Technology in Modern Teaching
}

\author{
Feng Bao \\ Daqing Petroleum Institute (Qinhuangdao Branch), Qinhuangdao 066004, China \\ E-mail: baofengqhd@sohu.com \\ Fengzhi Zhao \\ Daqing Petroleum Institute (Qinhuangdao Branch), Qinhuangdao 066004, China \\ E-mail: qhdcomputer@163.com
}

\begin{abstract}
Beginning with an exploration into the advantages and disadvantages of multimedia in current teaching, this article then elaborates on the proper ways to use this modern medium, hence offering reference for our modern teaching.

Keywords: Multimedia teaching, Teaching method, Courseware, Teaching experience

With the sharp rise of multimedia information technology giving birth to a series of high and new technological products, here has come an unprecedented revolution in the history of education technology. It has been acknowledged among Chinese educationalists that multimedia helps to improve class teaching efficiency. Among that, multimedia courseware, the principle force of computer-aided teaching, has also entered our classes. As an organic combination of teaching contents and teaching strategies, good courseware will attract students with its intuitionistic display of lecture contents through pictures, words, video and audio materials. Consequently, their enthusiasm for learning inspired greatly, students' predominant role in the teaching and learning process will be emphasized and the best teaching effect will be achieved.
\end{abstract}

\section{Advantages of Multimedia in Teaching}

\subsection{Greater Class Capacity}

It is much easier for teachers to explain as well as for students to understand those points beyond our expression by using dynamic demonstrations in class. Besides, for those courses emphasizing practical operating skills, multimedia is also helpful in expanding teaching capacity and improving teaching efficiency.

\subsection{Solving Focal and Difficult Points}

Unlimited in time and space, multimedia teaching will give more intuitionistic contents and effects, disintegrate the complex contents of knowledge, skills and information more completely, convey the teaching information, solve difficult points in teaching, display information in more variable ways and give prominence to focal points. For example, with the use of flash software, vivid and straightforward effects will be produced.

\subsection{Increasing Interactivity}

Interactivity should be the most distinctive characteristics of multimedia technology as well as the core of multimedia teaching. In a multimedia-aided class, teachers can have heuristic teaching by giving on-the-spot analysis and answers to students' feedback.

\subsection{Stimulating Students' Enthusiasm}

With multimedia providing for students comprehensive information with vocal, video, pictorial and literal effects, students will keep their attention longer and will have higher enthusiasm for learning. The use of multimedia in teaching is quite necessary, especially for some computer operation courses, in which only blackboard will not achieve desirable effects.

\subsection{Optimizing Teaching Contents}

When making their courseware, teachers tend to optimize their preparation for class. Therefore, it helps to improve teachers' teaching ability in a short time. In this sense, teaching with slides will help teachers a lot in grasping their courses as well as organizing their classes.

\section{Some Problems in the Use of Multimedia Courseware}

In spite of the widespread use of multimedia courseware nowadays, there are myths among teachers about its 
knowledge and use. They are mainly shown as follows:

\subsection{Following the Fashion}

Just in order to follow the fashion, many teachers use multimedia courseware in their teaching regardless of the features of their individual courses. Accordingly, a lot of courseware is simply composed of words, failing to attract students' attention. Besides, some teachers are confined to their courseware or pushed by the mouse, hence bonding up their as well as students' thoughts.

\subsection{Poor Priority Control of Multimedia Courseware}

Teachers are supposed to use multimedia flexibly to ensure reasonable teaching tempo and atmosphere. However, if multimedia courseware is used at improper situations, poor priority control will be caused.

\subsection{Lacking in circumstances}

The significance of interest for learning can be revealed in Einstein's quotation of "Interest is the best teacher". In fact, profound interest will stimulate learning motivation and encourage enthusiasm for learning effectively. However, our present multimedia teaching, due to its boring and onefold forms, fails to arouse students' learning interest.

\subsection{Lacking in Instances}

Teaching, coming from our life, applied to our life, displays some practical problems in our life to students with certain multimedia means and therefore, arouses their interest to explore knowledge. However, some multimedia courseware, only with simple literal introduction and lacking in practical instances, will surely fail to encourage students' enthusiasm for learning.

In short, it should be avoided to "refuse multimedia courseware when necessary or abuse it without purpose or plan". In our teaching process, multimedia courseware should be designed, developed and used properly in order to optimize our teaching contents and improve our class efficiency, hence exerting the real value of multimedia-aided teaching.

\section{The Development Tendency of Multimedia Teaching}

In 1990s, multimedia was used in the teacher-based combinatory teaching, while nowadays, with the combination of multimedia and network as well as the "two-basis" pattern (a pattern with both teachers' dominant role and students' important role as the only cognition subject), a new pattern of multimedia network teaching is coming into existence and developing rapidly. Now, we are able to compose a variety of multimedia teaching software, including instructive type, exercise type, testing type, simulative type, game type and so on. In addition, there is some other combinatory software, different parts of which can be used in different situations according to the actual demands in teaching.

Currently, multimedia software is mainly used in two ways: one is used in multimedia classrooms, in which digital projectors are employed to project image data on the screen and audio power amplifiers are used to increase the power of the audio signals output from the computer's audio card. This pattern is mainly suitable for teacher-based classes. For instance, in the teaching of Chinese as a foreign language in some universities, this pattern has been accepted by overseas students. The other is used in classrooms with computer network, in which the multimedia computers given for each student are combined by LAN and are equipped with network software and control software is installed on the teacher's computer. In such a classroom, with students participating in the teaching activity directly, students will study more voluntarily and more individually. In addition, teachers are able to control students' computers, to have direct dialogues with students, give individual instruction to them, answer their questions as well as conduct exercises or tests individually. Furthermore, if necessary, the computers can be connected to Internet to make full use of a variety of teaching materials in the teaching process. Therefore, it is safe to say that this pattern is more prospective for our modern education.

\section{Reasonable Use of Multimedia in Teaching}

First, teachers are expected to make their own multimedia courseware based on the teaching demands, the characteristics of teaching materials and students' actual situations. They can use it to further explain some concepts, to make it easier for students to understand some phenomena and rules and so on in their teaching process. In order to achieve that, teachers should know quite well about their courseware and operate them expertly, such as when to insert a picture, when to return to some point, when to give students some time for discussion and so on. Besides, teachers should adapt their courseware to students' mastery of knowledge. Although the courseware produced by professional software companies tends to be more beautiful and perfect in language, music, image and flash, it is not really fit for our class because it can't promote the communication between teachers and students, not to mention the interaction between them.

Second, courseware should be made according to actual situations. For example, slides, although fit for knowledge-based courses, are not fit for skill-based ones. For those instance-based courses, multimedia teaching is a good choice because it will display some practical examples in our real life to inspire students' enthusiasm for learning 
and exploration. Although many students wish to enjoy multimedia teaching, those teachers who are adopting this pattern should be informed to use flexible ways to put it across to students that they are acquiring skills to deal with real situations in the near future as well as learning knowledge. Accordingly, our teaching process shouldn't be confined to slides, but be closely related to different courses.

Third, various circumstances should be established to create active class atmosphere to encourage students' learning enthusiasm. In multimedia teaching, because of some intuitionistic information such as words, picture, flash, sound and image can be given synchronously, the teaching activity seems more concrete, more active, and tends to provide rich perception and ideas, and therefore, helps students to form abstract thinking instead of just perception. For instance, the "Bubble" part in Program Design of $\mathrm{C}$ is too boring for students to understand, nor is it easy for students to use in practice. What's worse, a lot of time will be wasted. So, if teachers demonstrate this part with multimedia courseware, students will understand it more easily and better.

Fourth, multimedia teaching should be combined with the traditional one to strengthen our teaching contents. Despite the significance of multimedia teaching, the traditional one shouldn't be abandoned. Greater emphasis should be put on the focal and difficult points in every class. Multimedia teaching seems quite necessary for those contents teachers feel it difficult to explain clearly to students. We will have brief but inclusive courseware with focused focal and difficult points.

It is "instruction" that an excellent teacher is characterized by and it is "understanding" that distinguishes an outstanding student from an average one. In same sense, multimedia courseware is just like an "electronic teacher", who should do a lot to instruct his students. Generally speaking, the multimedia software script composed by experienced teachers should include the following parts: the points difficult for students; the points to elaborate on; the points to omit or to mention briefly and the points for students to extend their thoughts.

\section{Conclusion}

To sum up, this article elaborates on the advantages and problems in our present multimedia teaching and comes up with some proper ways to conduct it. As for vocational colleges, both traditional teaching and multimedia teaching should be covered. With the development of our modern society, there are a greater variety of teaching targets. Therefore, it is a significant element for the improvement of teaching quality to employ multimedia in class design and it is a trend of teaching reforms to make better use of multimedia courseware.

\section{References}

Deng, Zemin. (2006). Teaching Design of Vocational Education. Bejing: China Railway Press.

Deng, Wenxin. (2007). Vocational Education. Vol. 501. pp5-7.

Zhao, Qi. (2007). Some Approaches to Improving Class Teaching with Multimedia Materials. [Online] Available: http://www.edu.cn/li_lun_yj_1652/20070905/t20070905_252377_1.shtml. (September 5th, 2007).

Zhu, Weimin. The Application of Multimedia Materials in Class Teaching. A Collection of Theses of Electronic Teaching Center in Beijing Language and Culture University. [Online] Available: http://www.blcu.edu.cn/djzx/index.htm 